\title{
Nouvelle station de Pteris vittata L. (Pteridaceae) en Numidie (Algérie orientale)
}

\author{
Tarek Hamel ${ }^{1,2}$, Gérard de Bélair ${ }^{3},{\text { Abderachid Slimani }{ }^{1}, \text { Lamia Boutabia }^{4} \text { \& Salah Telailia }}^{4}$ \\ ${ }^{1}$ Laboratoire de Biologie Végétale et Environnement, Département de Biologie, Faculté des Sciences, Université Badji \\ Mokhtar, 12 rue Hassi Beida, 23000 Annaba, Algérie. \\ 'Laboratoire Sols et Développement Durable, Département de Biologie, Faculté des Sciences, Université Badji Mokhtar, 23 \\ 000 Annaba, Algérie \\ ${ }^{3}$ Université Badji Mokhtar, 12 rue Hassi Beida, 23000 Annaba, Algérie \\ ${ }^{4}$ Laboratoire Agriculture et Fonctionnement des Ecosystèmes, Faculté des Sciences de la Nature et de la Vie, Université \\ Chadli Bendjedid, BP 73, 36000 El Tarf, Algérie
}

\author{
Correspondencia \\ Tarek Hamel \\ email: tarek_hamel@yahoo.fr \\ Recibido: 5 marzo 2019 \\ Aceptado: 7 noviembre 2019 \\ Publicado on-line: 20 abril 2020 \\ Editado por: A. Enrique Salvo Tierra
}

\begin{abstract}
Résumé
Les auteurs signalent une observation de Pteris vittata L. dans la Numidie algérienne. Cette découverte permet de faire le point sur sa répartition en Algérie.
\end{abstract}

Mots clés: Pteris vittata, Algérie, nouvelle répartition, rare.

\begin{abstract}
New station of Pteris vittata L. (Pteridaceae) in Numidia (Eastern Algeria)
\end{abstract}

The authors report an observation of Pteris vittata L. in Numidia (Eastern Algeria). This discovery makes it possible to take stock of its distribution in Algeria.

Key words: Pteris vittata, Algeria, new distribution, rare.
Le nombre de taxons de Ptéridophytes acceptés en Afrique du Nord est de 114; dont 58 taxons sont présents en Algérie (Dobignard \& Chatelain, 2010). La Numidie $\left(\mathrm{K}_{3}\right.$ au sens de la subdivision biogéographique proposée par Quézel \& Santa, 1962-1963) recèle $60 \%$ de ces espèces (Louhi-Haou, 2014).

Pteris vittata $\mathrm{L}$. ou ptéride à longues feuilles est un taxon subcosmopolite assez répandu sur le sud de la Méditerranée jusqu'en Afrique subsaharienne en passant par le Cap et à l'est par le Moyen-Orient, la Russie et le sous-continent indien jusqu'en ExtrêmeOrient et au sud en passant par le Sud-Est de l'Asie jusqu'en Australie. Elle a apparemment été introduite dans un certain nombre de groupes d'îles de l'océan Pacifique, notamment la Nouvelle-Zélande, I'Amérique du Nord, l'Amérique centrale et l'Amérique du Sud (Blanca et al., 2009 ; Hassler \& Schmidt, 2011). Cette fougère avait été évaluée comme moins préoccupante (LC) à l'échelle mondiale par I'UICN (2020).

En Algérie, le genre Pteris L. est représenté par $P$. cretica $L$. qui est une espèce rarissime selon Quézel
\& Santa (1962-1963) et $P$. vittata L. espèce très localisée observée sur les rochers suintants et les ravins humides sur le littoral et dans les montagnes de petite Kabylie $\left(K_{2}\right)$, l'Atlas Tellien $\left(A_{2}\right)$ et les Andalouses ( $\mathrm{O}_{1}$ ) (Quézel \& Santa, 1962).

Cette dernière espèce ne semble pas avoir été signalée en Numidie (Maire, 1952 ; Quézel \& Santa, 1962-1963 ; Haou et al., 2011 ; Hamel et al., 2017 ; Hamel et al., 2018). C'est à proximité de la plage Saint Louis que nous l'avons trouvée sur la bordure d'un ruisseau sous Salix pedicellata Desf. (Saule pédicellé) et Alnus glutinosa (L.) Gaertn. (Aulne glutineux). Un échantillon de la plante a été récolté et déposé dans l'herbier de Gérard de Bélair [Nouveau taxon pour la Numidie, $\left(36^{\circ} 54^{\prime} 24,22^{\prime} \mathrm{N} ; 7^{\circ} 11^{\prime} 71^{\prime \prime} \mathrm{E}\right)$, $6 \mathrm{~m}$, ripisylve, 19. II. 2016. Herbier GDB, $\mathrm{n}^{\circ} 111 \_36$ http.//gdebelair.com/imascan/111_36.jpg] (Fig. 1).

La présence de cette fougère en bordure des sites historiques en $\mathrm{K}_{3}$ vient donc compléter sa présence en Tunisie sur la frontière algérienne à Tabarka (Guénod et al., 1954).

La population de l'espèce (Fig. 2) observée est très réduite, elle s'élève à cinq pieds isolés avec une 


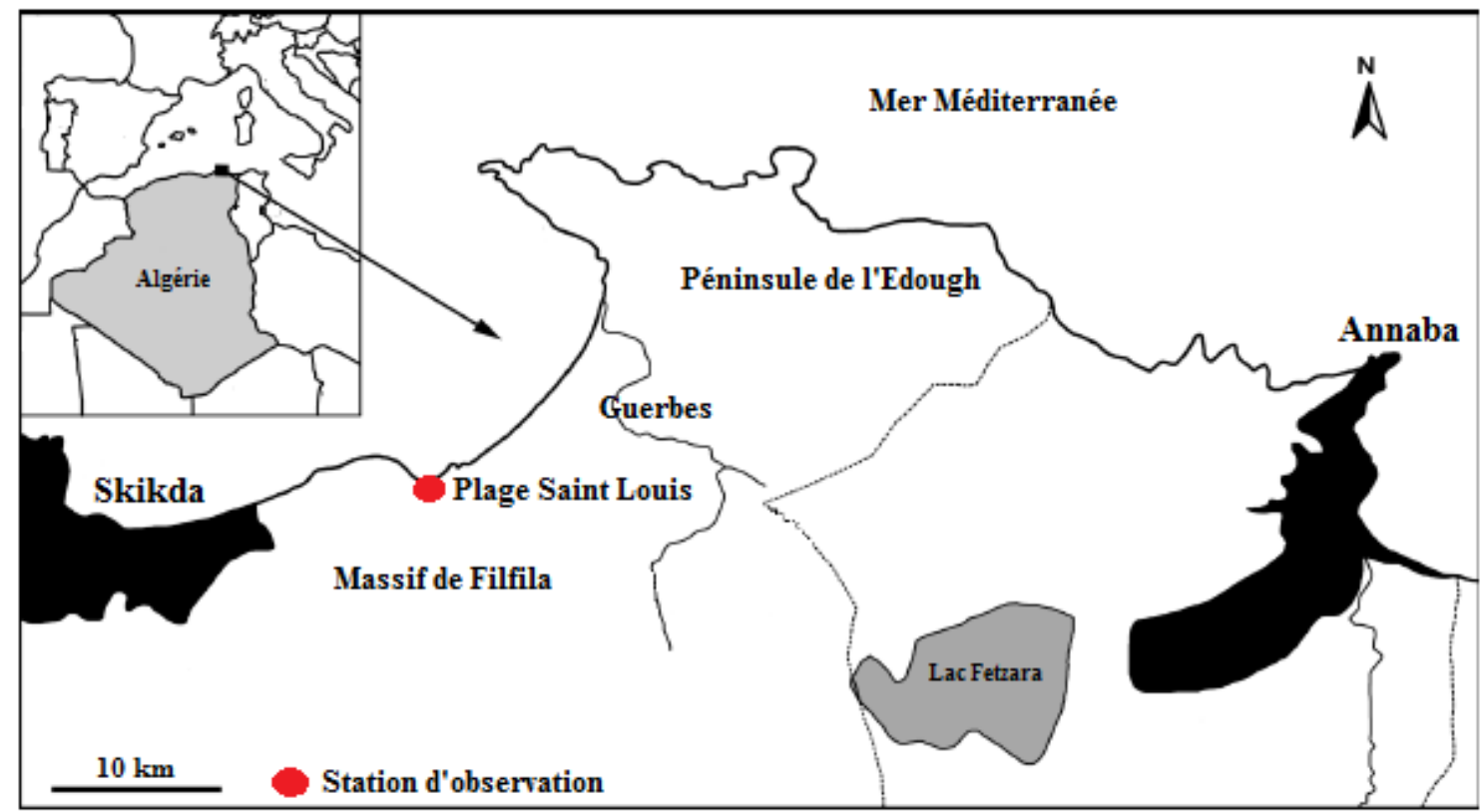

Figure 1. Localité de la station d'observation.

Figure 1. Locality of the observation station.

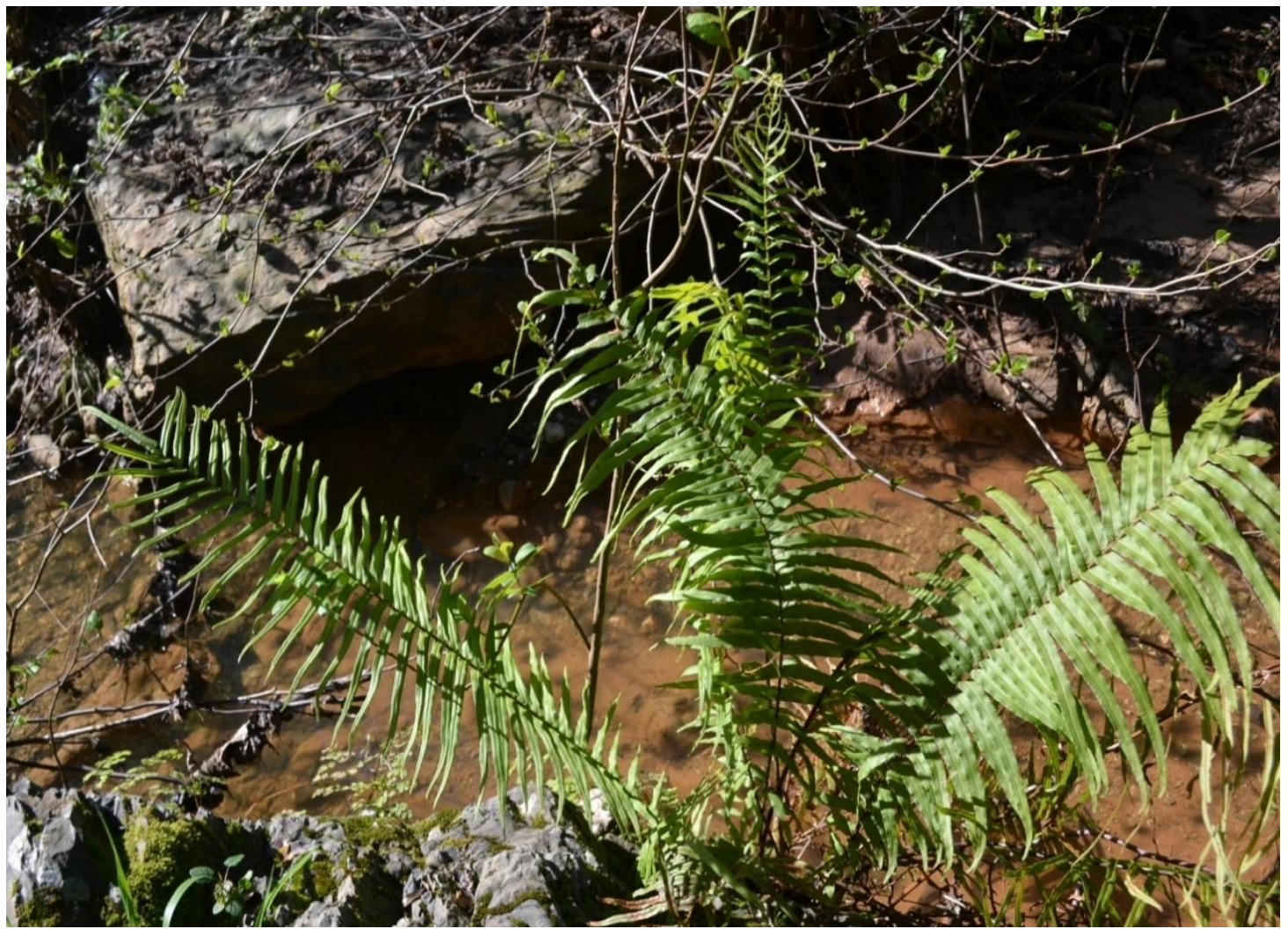

Figure 2. Pteris vittata L. dans son habitat (Cliché HAMEL T. plage Saint Louis, 19/02/2016).

Figure 2. Pteris vittata L. in its habitat (Cliche HAMEL T. Saint Louis beach, 19/02/2016).

faible régénération à cause de la fréquentation de la plage dans la période estivale.

La présence de nombreux ruisseaux difficiles d'accès, nous laisse penser que d'autres populations de ladite fougère peuvent exister au niveau des contreforts de Filfila demeurés inexplorés sur le plan botanique.
En effet, ils sont partie prenante du point-chaud de biodiversité végétale nommé "Kabylies-NumidieKroumirie" (Véla \& Benhouhou, 2007). Aujourd'hui, l'accessibilité à plusieurs zones de ce point-chaud s'est nettement amélioré par rapport au milieu du 20 éme siècle, et plusieurs espèces (spontanées) nouvelles pour l'Algérie ont d'ores-et-déjà été 
découvertes dans les régions proches de la station d'observation notamment à Guerbes et à la péninsule de l'Edough: Nymphoides peltata (Gmel.) O. Kuntze (de Bélair \& Véla, 2011), Malva arborea (L.) Webb et Berthel. (Amari et al., 2015), Romulea leichtliniana Halácsy et Sixalix farinosa (Coss.) Greuter et Burdet (Hamel \& Boulemtafes, 2017a ; Boulemtafes et al., 2018), Soleirolia soleirolii (Req.) Dandy (Hamel \& Boulemtafes, 2017b). A l'opposé de la zone occidentale de la plage Saint Louis n'avait pas encore fait l'objet d'investigations poussées.

En revanche, la grande rareté de Pteris vittata au Maghreb, met en avant sa valeur patrimoniale et l'importance de la station d'observation pour la conservation de cette espèce. Ainsi, le massif de Filfila peut constituer une mini zone importante pour les plantes (ZIP) bien individualisée faisant continuité géographique et écologique de la ZIP de Guerbes déjà identifiée plus au sud (Benhouhou et al., 2018).

\section{Bibliographie}

Amari, D., Moulai, R., de Bélair, G. \& Véla, E. (2015). Nouvelles observations de Malva arborea (L.) Webb et Berthel. sur le littoral algérien. Acta Botanica Malacitana, 40, 213-216.

Benhouhou, S., Yahi, N. \& Véla, E. (2018). Key Biodiversity Areas (KBAs) for plants in the Mediterranean region, chapter 3, IUCN, (pp. 5360 ).

Blanca, G., Cabezudo, B., Cueto, M., Lopez, C.F. \& Torres, C.M. (2009). Flora Vasculair de Andalucía Oriental. Tome 1. Consejería de Medio Ambiente. Junta de Andalucía.

Boulemtafes, A., Hame,I T., de Bélair, G. \& Véla, E. (2018). Nouvelles données sur la distribution et l'écologie de seize taxons végétaux du littoral de la péninsule de l'Edough (Nord-Est algérien). Bulletin Society Linnean of Provence, 69, 59-76.

de Bélair, G. \& Véla, E. (2011). Découverte de Nymphoides peltata (Gmel) O. Kuntze (Menyanthaceae) en Afrique du Nord (Algérie). Poiretia, 3, 1-7.

Dobignard, A. \& Chatelain, C. (2010-2013). Index synonymique de la flore d'Afrique du Nord. 5 Tomes. Eds des Conservatoire et Jardin Botanique de Genève.
Guénod, A., Pottier-Alapetite, G. \& Labbe, A. (1954). Flore de la Tunisie, Cryptogames Vasculaires, Gymnospermes et Monocotylédones, Imprimerie S.E.F.A.N.

Hamel, T. \& Boulemtafes, A. (2017a). Nouvelle station de Sixalix farinosa (Coss.) Greuter et Burdet dans la péninsule de l'Edough (Nord-Est algérien). Bulletin Society Linnean of Provence, $68,1-9$.

Hamel, T. \& Boulemtafes, A. (2017b). Découverte d'une endémique tyrrhénienne Soleirolia soleirolii (Urticaceae) en Algérie (Afrique du Nord). Flora Mediterranea, 27, 185-193.

Hamel, T., Slimani, AR., Madoui, B.E.M. \& Boulemtafes, A. (2017). Pteridophytes of Edough peninsula (North East Algeria). International Journal of Research in Ayareda and Pharmacy, 8 (1), 92-96.

Hamel, T., Boulemtafes, A. \& Bellili, A.M. (2018). Inventaire des ptéridophytes dans le Parc National d'El Kala (Algérie orientale). Acta Botanica Malacitana, 43, 31-42.

Haou, S., de Bélair, G. \& Viane, R.L.L. (2011). Inventory of the ferns (filicopsida) of Numidia's (North-Eastern Algeria). International Journal of Biodiversity and Conservation, 3 (6), 206-223.

Hassler, M. \& Schmidt, B. (2011). Checklist of ferns and lycophytes of the world. [en ligne]. https://worldplants.webarchiv.kit.edu/ferns/statisti cs.php [Accès le 07 février 2019].

Louhi-Haou, S. (2014). Ecologie des ptéridophytes en Numidie (Nord Est de l'Algérie). Thèse de Doctorat en Ecologie Végétale, Université Badji Mokhtar Annaba, Algérie.

Maire, R. (1952). Flore de l'Afrique du Nord. Volume 1. Ed. Le chevalier Paris.

Quézel, P. \& Santa, S. (1962-1963). Nouvelle flore de l'Algérie et des Régions Désertiques Méridionales. Paris: CNRS. Tome 1, 558 p. Tom 2, 603p.

UICN (2020). Liste rouge de I'UICN des espèces menacées. Version 2020. http://www.iucnredlist.org. [Accès le 07 février 2019].

Véla, E. \& Benhouhou, S. (2007). Évaluation d'un nouveau point chaud de biodiversité végétale dans le bassin méditerranéen (Afrique du Nord). Comtes Rendus Biologies, 330, 589-605. 